\title{
Understanding the Implementation of Hierarchical Medical System Policy: An Interrupted Time Series Analysis of a Pilot Scheme in China
}

Huajie Hu

Peking University

\section{Ruilin Wang}

Peking University

Huangqianyu Li

Peking University

Sheng Han

Peking University

\section{Peng Shen}

Department of chronic disease and' health promotion

\section{Hongbo Lin}

Department of chronic disease and health promotion

Xiaodong Guan ( $\nabla$ guanxiaodong@pku.edu.cn )

Peking University School of Pharmaceutical Sciences https://orcid.org/0000-0002-1290-3827

Luwen Shi

Peking University School of Pharmaceutical Sciences

\section{Research}

Keywords: Hierarchical medical system, Primary care, Integrated care, Social network analysis, Medical consortium

Posted Date: December 13th, 2021

DOI: https://doi.org/10.21203/rs.3.rs-1134925/v1

License: (c) (i) This work is licensed under a Creative Commons Attribution 4.0 International License. Read Full License 


\section{Abstract}

\section{Background}

The Chinese healthcare system faces a dilemma between its hospital-centric approach to healthcare delivery and a rapidly aging population that requires strong primary care. To improve system efficiency and continuity of care, the Hierarchical Medical System (HMS) policy package was implemented in 2015 in Zhejiang province, China. This paper investigated the impact of HMS on the local healthcare system.

\section{Methods}

We conducted a repeated cross-sectional study with quarterly data collected between 2010 and 2018 from Yinzhou district, Ningbo. The data was analyzed with an interrupted time series (ITS) design to assess the impact of HMS on the changes of three outcome variables: primary care physicians (PCPs) patient encounter ratio (i.e., the mean quarterly number of patient encounters of PCPs divided by that of all other physicians), PCP degree ratio (i.e., the mean degree of PCPs divided by that of all other physicians), PCP betweenness centrality ratio (i.e., the mean betweenness centrality of PCPs divided by that of all other physicians).

\section{Results}

272,267 patients visited doctors for hypertension between 2010 and 2018. Compared to the counterfactual in the fourth quarter of 2018 , the PCP patient encounter ratio rose by $42.7 \%$ (95\% Cl: $27.1-58.2, p<0.001)$, the PCP degree ratio increased by $23.6 \%(95 \% \mathrm{Cl}: 8.6-38.5, \mathrm{p}<0.01)$, and the PCP betweenness centrality ratio grew by $129.4 \%(95 \% \mathrm{Cl}$ : $87.1-171.7, \mathrm{p}<0.001)$.

\section{Conclusions}

The HMS policy can incentivize patients to visit primary care facilities and enhance the centrality of PCPs within their professional network. Local policymakers should sustain HMS policy efforts to obtain long-term and large-scale benefits.

\section{Contributions To The Literature}

- To our best knowledge, this is the first quasi-experimental study to evaluate the effectiveness of hierarchical medical system (HMS) policy in a middle-income country.

- The HMS policy of Yinzhou model is effective to incentivize patients to visit primary care facilities and enhance the centrality of primary care physicians within their professional networks. The findings of this study may contain important value for building integrated care delivery networks

- This study adopts social network analysis to investigate the impact of health care reform, which provides a reference for health policy evaluation from a methodological standpoint.

\section{Background}

Healthcare systems worldwide are combatting challenges emerging from the epidemiological transition from acute infectious diseases to chronic non-communicable diseases and an accelerated population aging process.[1-5] Focusing on hospital-based and life-threatening conditions, medical services in many countries do not match with the increasing demand for controlling non-communicable diseases.[6-8] Therefore, the World Health Organization (WHO) considered referral a core part of a comprehensive health care system,[9] suggested that primary care should be 
strengthened to provide effective and efficient care, [10] and proposed that a framework on integrated people-centered health services should be adopted in all countries.[6] Health services in most western countries are delivered in a hierarchical medical system (HMS) and through the mandatory gatekeeping mechanism which involves initial diagnoses at primary care facilities (PCFs) and obligatory two-way referrals among hospitals.[11, 12] With the fundamental HMS and the backbone of a strong primary care system, many developed countries have attempted to establish chronic care models to provide integrated care, which has proved to be effective in improving care and reducing costs. $[10,13]$ While experience from developed countries provides useful references, the routine to rebuild the healthcare delivery system is highly context-specific, and there is limited evidence in low- and middle-income countries (LMIC).[4, 14] Patients in LMIC often bypass primary care facilities and $53 \%$ patients seek care directly at tertiary hospitals, suggesting lower level of efficiency.[15, 16]

As in many developing countries, the Chinese medical system does not work as intended and faces many problems. The current health care system in China is an inverted pyramid, which is not sustainable.[17] Approximately $82 \%$ of resources were concentrated at tertiary hospitals and secondary, and only $18 \%$ at primary care facilities.[18] Such unbalanced distribution of resources results in extremely high patient flows to large hospitals with underutilization of primary care, which is inefficient and costly(Figure 1).[11, 19] Additionally, the current hospital-centered and treatmentdominated healthcare systems in China are facing considerable challenges of fragmented care, with little effective collaboration among institutions at different grades.[1] Fragmentation of services negatively impacts patient satisfaction and further aggravated health inequity among residents.[10]

To optimize residents' current healthcare-seeking behaviors and improve the efficiency of healthcare delivery, the Chinese government introduced the HMS policy package in 2014[20]. Under this policy package, different levels of hospitals have a clear division of labor and coordinate to provide continuous, comprehensive and integrated care to individuals. By the end of 2016, there were four municipalities and 266 cities that have launched HMS, covering $88.1 \%$ of mainland China.[20] Despite the importance and the expansion of the HMS at the national level, we know very little about the impact of the HMS policy in China. Previous studies focused on cognition,[21] factors, [20]theoretical analysis and health-seeking behavior of residents,[22] but empirical research is scarce. Whether the HMS has been successfully implemented in these pilot cities is still unascertained. Experience from Yinzhou, one of the earliest pilot regions, has essential policy implications. We make an initial contribution to filling the gaps above by answering the following questions: whether does the HMS policy successfully redirect the patient flows to primary care facilities in Yinzhou? how does this reform impact the role of PCPs in the local health care system?

\section{Methods}

\section{Study Setting}

We conducted a retrospective study in Ningbo, a coastal city in southeast China. As the largest district in Ningbo, Yinzhou had a population of 1.34 million in 2018.[23] In 2014, Yinzhou introduced its HMS policy package, including three intertwined actions (figure 1): (1) Residents were encouraged to sign a one-year contract with a PCP to appoint the PCP as the resident's family doctor, who would act as a gatekeeper of specialist care. The resident shall visit the PCP for an initial diagnosis, and visit a specialist doctor at higher-tier hospitals with a referral letter from the PCP. (2) Health facilities across all tiers shall form hospital networks to provide coordinated care. Each network shall include one recognized tertiary hospital and multiple secondary hospitals and PCFs. (3) Patients were financially incentivized to utilize primary care through a gradient reimbursement scheme, differentiating reimbursement rates between hospital visits with or without a PCP referral. (Appendix 1). More details about the Yinzhou model are presented in Appendix 2. 


\section{Data Sources}

We collected data from the Yinzhou Health Information System (YHIS), whose registration covered 98\% of permanent residents in Yinzhou district since 2009. YHIS integrated data from different administrative databases encompassing population census, residents' electronic medical records, hospital information system, health insurance, disease surveillance and management, chronic diseases recording system, health checks and death reports. [24] We retained patient encounter data from patients who visited physicians for hypertension between January 12010 and December 312018 as the local HMS policy package had a specific focus on chronic disease management. Eligible patient encounter data was identified based on the diagnosis shown on the records of outpatients or inpatients (10th revision of the International Classification of Diseases [ICD-10]: I10, I11, I12, I13, I15). All information used was de-identified and this study was exempt from review by the Ethics Committee of Peking University Health Science Centre, Beijing, China.

\section{Study Design}

Using quarterly data of patient encounters, we investigated the impact of the HMS policy on the utilization of PCFs and the role of PCPs within their professional network. We conducted an interrupted time series (ITS) analysis,[25] with the one-year intervention period defined as the period from the issue date of the HMS policy (the fourth quarter of 2014) to its full implementation (the third quarter of 2015). For each variable of interest, we conducted a separate analysis on all patient observations, with the 36 quarterly time points as units of analysis. Observed results were compared with counterfactual scenarios based on extrapolation of pre-HMS trends, which simulated likely trajectories in the absence of HMS. We estimated the difference between the observed results from the regression models and the expected results stemming from the counterfactual scenarios at the end of the study period (i.e., 2018Q4). There were no other policies or major regulations which might affect the impact of the HMS during the study period.

\section{Outcome Measures}

Patient encounter

The HMS package in Yinzhou aimed to redirect patient flows from secondary and tertiary hospitals to PCFs. Changes in patient flows can be observed by changes in patient encounters of specialist doctors and PCPs. We thus calculated the number of quarterly patient encounters of every physician, including PCPs. To measure the relative patient volume of PCPs, we calculated PCP patient encounter ratio, which was defined as the mean number of quarterly patient encounters of PCPs divided by that of all other physicians.

Network metrics

We also conducted a Social Network Analysis and calculated network metrics to evaluate of the HMS policy's impact on the practices of local healthcare professionals. Literature suggested that patient-sharing identified using administrative data was indicative of critical physician relationships such as physician groups providing services within the same network. Therefore, we defined physician dyads based on such patient-sharing relationships identified through administrative data. [26] [27-30]

We first identified and extracted claim data of physician dyads in Yinzhou (Figure 2 panel A). This data subset was then transformed into a physician-physician network (Figure 2 panel B). The weight of each physician-physician tie equaled to the number of patients they shared. To identify a valid physician-physician network, we chose different thresholds (i.e., the number of patients shared by each pair of physicians) to test the model. We then calculated two classical network metrics, degree and betweenness centrality, to evaluate the structural characteristics and network 
position of a physician.[31-33] In this study, degree revealed the activity and popularity of a physician, reflecting the extent to which he/she coordinated with others in delivering health services; betweenness centrality was interpreted as the relative importance of a physician within the network, indicating his/her potential influence over healthcare delivery.[34, 35] The illustrative model of these two measures is available in Figure 2 (Panel C and D).

Degree is defined as the number of other physicians connected to a given physician through the sharing of patients. A physician with a higher degree may share patients with a broader array of physicians and be considered more active in the network. Degree ranges from 0 to $N-1$, and is given by

$$
\operatorname{degree}(i)=\sum_{i \neq j} d_{i j}(1)
$$

where $\mathrm{d}_{\mathrm{ij}}$ denotes the link between node $\mathrm{i}$ and $\mathrm{j}$. Degree only estimates direct connections that a node has and excludes indirect connections which are expansive in physician networks. We use PCP degree ratio to measure on average, how active or popular PCPs are compared to other physicians by calculating the ratio of the mean degree of PCPs versus all other physicians in the network.[27, 36, 37]

Unlike degree, which captures only the immediate links, betweenness centrality measures both direct and indirect connections of a node to quantify the structural centrality of the node in the network. The betweenness centrality of a physician is proportional to the frequency of this physician lying on the shortest paths when screening for all shortest paths from every physician to every other physician in the network.[32] That is, if a physician has a higher betweenness centrality (i.e., lies on more shortest paths), he/she is considered more central in the network and more likely to hold influence over information flow and practice uptake. The most commonly used betweenness centrality is defined as

$$
\operatorname{betweennesscentrality}(i)=\sum_{i \neq v \neq j} \frac{g(v, j \mid \imath)}{g(V, j)}(2)
$$

where $g(v, j \mid i)$ is the total number of the shortest paths between $v$ and $j$ that pass through $i$, and $g(v, j)$ is the total number of the shortest paths between $v$ and $j$ (regardless of whether they pass through $\mathrm{i}$ ). For the comparison across graphs, normalization is performed by multiplying the raw betweenness centrality by $2 /\left(n^{2}-3 n+2\right)$, where $n$ is the number of nodes in the graph. To calculate the relative betweenness centrality for PCPs, we calculated the PCP betweenness centrality ratio (i.e., the mean betweenness centrality of the primary care physicians divided by the mean betweenness centrality of all other physicians in the network). [27, 36, 37]

\section{Statistical Methods}

We conducted an ITS ordinary least squares regression using Newey-West standard errors to accommodate for serial autocorrelation and adjusted for autocorrelation heteroskedasticity in time series up to four lags.[38] We used a twosided hypothesis test with a threshold for decision-making of $p=0.01$. In the sensitivity analysis, we used the occurrence time of the HMS policy to assess whether the impact measured could be attributed to HMS implementation.[39] All network metrics were calculated using the "igraph" package (version 1.2.6) in R (version 4.0.3) and statistical analyses were conducted using Stata 15.0.

\section{Results}

272,267 patients saw doctors for hypertension between 2010 and 2018 and the total number of patient encounters was 9,270,974. Among 42 sample hospitals, there were 34 PCFs, three secondary hospitals, and five tertiary hospitals. 
Stratified by tier classification of their site of practice, 2,142 physicians practiced at PCFs, 243 practiced at secondary hospitals, and 1,531 at tertiary hospitals.

We analyzed quarterly data of 45,464 patient observations by physicians over 36 time points between January 2010 and December 2018. At each time point, over $94.3 \%$ of physicians had at least one patient-sharing connection with others. Table 1 shows descriptive statistics comparing the main outcome variables of physicians at three time points: 2010Q1, 2015Q3, 2018Q4. In total, the mean number of physicians' quarterly patient encounters increased from 218.5 to 244.4 whilst the average degree of physicians decreased from 29.3 to 27.9 after the introduction of the HMS policy. Stratified by tiers, all outcome measures of PCPs scored lower than those of physicians at higher-tier hospitals at $2010 \mathrm{Q} 1$ but the situation reversed at 2018Q4 ( $<0.001$ in all cases). 
Table 1

Descriptive statistics of patient encounters, degree, and betweenness centrality of physician observations.

\begin{tabular}{|c|c|c|c|c|c|c|c|c|c|c|}
\hline \multirow[t]{2}{*}{ Outcomes } & \multirow[t]{2}{*}{$\mathbf{N}^{+}$} & \multicolumn{3}{|c|}{ 2010Q1 } & \multicolumn{3}{|c|}{ 2015Q3 } & \multicolumn{3}{|c|}{ 2018Q4 } \\
\hline & & $n^{\square}$ & mean & $P^{\prime}$ & $n^{\square}$ & mean & $P^{\prime}$ & $n^{\square}$ & mean & $P^{\prime}$ \\
\hline $\begin{array}{l}\text { Patient } \\
\text { encounters }\end{array}$ & 45464 & 894 & 103.103 & & 1344 & 218.478 & & 1295 & 244.361 & \\
\hline I & 32159 & 685 & 112.073 & \multirow[t]{3}{*}{$<0.001$} & 946 & 276.633 & \multirow[t]{3}{*}{$<0.001$} & 792 & 364.936 & \multirow[t]{3}{*}{$<0.001$} \\
\hline II & 2407 & 36 & 141.611 & & 54 & 360.296 & & 70 & 286.814 & \\
\hline III & 10898 & 173 & 59.572 & & 344 & 36.291 & & 433 & 16.956 & \\
\hline Ratio $\ddagger$ & & & 1.521 & & & 3.447 & & & 6.695 & \\
\hline Degree & 45464 & 894 & 20.611 & & 1344 & 29.331 & & 1295 & 27.942 & \\
\hline 1 & 32159 & 685 & 19.509 & \multirow[t]{3}{*}{$<0.001$} & 946 & 31.258 & \multirow[t]{3}{*}{$<0.001$} & 792 & 33.999 & \multirow[t]{3}{*}{$<0.001$} \\
\hline II & 2407 & 36 & 22.313 & & 54 & 44.283 & & 70 & 36.130 & \\
\hline III & 10898 & 173 & 24.994 & & 344 & 20.718 & & 433 & 14.203 & \\
\hline Ratio ${ }^{\ddagger}$ & & & 0.795 & & & 1.289 & & & 1.939 & \\
\hline $\begin{array}{l}\text { Betweenness } \\
\text { centrality }\end{array}$ & 45464 & 894 & 0.002 & & 1344 & 0.002 & & 1295 & 0.002 & \\
\hline I & 32159 & 685 & 0.002 & \multirow[t]{3}{*}{$<0.001$} & 946 & 0.001 & \multirow[t]{3}{*}{$<0.001$} & 792 & 0.002 & \multirow[t]{3}{*}{$<0.001$} \\
\hline II & 2407 & 36 & 0.003 & & 54 & 0.002 & & 70 & 0.002 & \\
\hline III & 10898 & 173 & 0.005 & & 344 & 0.002 & & 433 & 0.001 & \\
\hline Ratio $\ddagger$ & & & 0.400 & & & 0.712 & & & 1.481 & \\
\hline
\end{tabular}

t: $\mathrm{N}$ represents the total number of observations over all time points included in the analyses

邓: $\mathrm{n}$ represents the number of observations at each time point

ı : P value from Kruskal-Wallis test comparing outcomes at different hospital grades

‡: PCP patient encounter ratio (i.e., the mean number of quarterly patient encounters of primary care physicians divided by that of all other physicians), PCP degree ratio (i.e., the mean degree of PCPs divided by the mean degree of all other physicians), PCP betweenness centrality ratio (i.e., the mean betweenness centrality of PCPs divided by the mean betweenness centrality of all other physicians)

Hospital grade: I, Primary care facilities; II, Secondary Hospitals; III, Tertiary Hospitals

Table 2 compares the outcome measures with counterfactual scenarios in which pre-HMS trends were extrapolated.

Figure 3 shows the trends before and after the implementation of the HMS policy for the three outcome measures. All outcome measures had experienced small increases before the announcement of the HMS (the baseline trend for PCP patient encounters ratio, PCP degree ratio, and PCP betweenness centrality ratio were $0.107,0.041$, and 0.035 ,

respectively, $p<0.001)$. Despite showing little rapid change shortly following the full implementation of HMS ( $p>0.05$ for all post-implementation level change), the increase of all outcome measures accelerated with time ( $p<0.01$ for all post-implementation trend change). This accelerated increase finally resulted in a $42.7 \%(95 \% \mathrm{Cl}: 27.1-58.2)$ growth in 
PCP patient encounter ratio, a 23.6\% (95\%Cl: 8.6-38.5) growth in PCP degree ratio, and a 129.4\% (95\% Cl: 87.1-171.7) growth in PCP betweenness centrality ratio at 2018Q4 as compared with the counterfactual. The impacts of the HMS policy on all outcome measures were robust to different thresholds (Table 2, Appendix 3). In the sensitivity analysis, the estimated effect of the HMS policy in the two years prior to the occurrence time of the HMS policy was insignificant (Appendix 4). 
Table 2

Results of ITS analyses of the impacts of the hierarchical Medical System (HMS) policy on PCP patient encounter ratio, PCP degree ratio, and PCP betweenness centrality ratio.

\begin{tabular}{|c|c|c|c|c|c|c|c|}
\hline Outcomes & $\begin{array}{l}\text { Threshold } \\
\text { (No. of } \\
\text { shared } \\
\text { patients) }\end{array}$ & $\begin{array}{l}\text { Baseline } \\
\text { level }\end{array}$ & $\begin{array}{l}\text { Baseline } \\
\text { trend }\end{array}$ & $\begin{array}{l}\text { Post- } \\
\text { implementation } \\
\text { level change }\end{array}$ & $\begin{array}{l}\text { Post- } \\
\text { implementation } \\
\text { trend change }\end{array}$ & $\begin{array}{l}\text { Absolute } \\
\text { difference } \\
\text { in } \\
2018 Q 4 \\
(95 \% \mathrm{Cl})^{\dagger}\end{array}$ & $\begin{array}{l}\text { Percentage } \\
\text { change in } \\
2018 \mathrm{Q} 4 \\
(95 \% \mathrm{Cl})^{\ddagger}\end{array}$ \\
\hline
\end{tabular}

PCP patient encounters ratio

\begin{tabular}{|c|c|c|c|c|c|}
\hline $\begin{array}{l}1 \\
433^{* \star *}\end{array}$ & $\begin{array}{l}0 . \\
107 * * *\end{array}$ & -0.990 & $0.250 \star \star \star *$ & 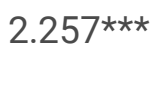 & 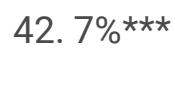 \\
\hline & & & & $\begin{array}{l}(1.431 \text { to } \\
\text { 3. } 083)\end{array}$ & $\begin{array}{l}(27.1 \text { to } \\
58.2)\end{array}$ \\
\hline
\end{tabular}

PCP degree ratio

\begin{tabular}{|c|c|c|c|c|c|c|}
\hline $\begin{array}{l}\geq \text { one } \\
\text { patient }\end{array}$ & $0.813^{\star \star \star}$ & $0.023^{\star \star \star}$ & -0.041 & $0.033^{\star \star}$ & $0.383^{\star \star}$ & $23.6 \%$ ** \\
\hline & & & & & $\begin{array}{l}(0.155 \text { to } \\
0.611)\end{array}$ & $\begin{array}{l}(8.6 \text { to } \\
38.5)\end{array}$ \\
\hline \multirow{2}{*}{$\begin{array}{l}\geq \text { three } \\
\text { patients }\end{array}$} & $1.142^{\star \star \star}$ & $0.055^{\star \star \star}$ & -0.242 & $0.091 * * *$ & $0.942 * \star \star$ & $30.3 \% * * *$ \\
\hline & & & & & $\begin{array}{l}\text { (0.510 to } \\
1.374)\end{array}$ & $\begin{array}{l}\text { (14.4 to } \\
46.1)\end{array}$ \\
\hline \multirow[t]{2}{*}{$\begin{array}{l}\geq \text { five } \\
\text { patients }\end{array}$} & $1.243^{\star \star *}$ & $0.065^{\star \star \star}$ & -0.286 & $0.124^{\star \star \star}$ & $1.320 * \star \star$ & $36.9 \% * \star \star *$ \\
\hline & & & & & $\begin{array}{l}(0.626 \text { to } \\
2.014)\end{array}$ & $\begin{array}{l}(14.2 \text { to } \\
59.6)\end{array}$ \\
\hline
\end{tabular}

PCP betweenness centrality ratio

\begin{tabular}{|c|c|c|c|c|c|c|}
\hline$\geq$ one & $0.423^{\star \star \star}$ & $0.007 * \star \star$ & 0.035 & $0.063^{* \star *}$ & $0.860 * \star \star$ & $129.4 \% * * *$ \\
\hline & & & & & $\begin{array}{l}(0.679 \text { to } \\
1.041)\end{array}$ & $\begin{array}{l}\text { (87.1 to } \\
171.7)\end{array}$ \\
\hline \multirow{2}{*}{$\begin{array}{l}\geq \text { three } \\
\text { patients }\end{array}$} & $0.539 * \star \star$ & $0.015^{\star \star \star}$ & 0.226 & $0.052 * \star$ & $0.908^{\star \star *}$ & 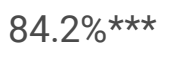 \\
\hline & & & & & $\begin{array}{l}(0.605 \text { to } \\
1.211)\end{array}$ & $\begin{array}{l}\text { (54.7 to } \\
113.6)\end{array}$ \\
\hline $\begin{array}{l}\geq \text { five } \\
\text { patients }\end{array}$ & $0.628 * \star \star$ & $0.040 * \star \star$ & -0.527 & $0.306 * \star \star$ & $3.454 * \star \star$ & $168.3 \%$ *** \\
\hline
\end{tabular}

†: Absolute difference in 2018Q4, the difference between the observed outcome and the counterfactual (extrapolation of pre-announcement trend)

$¥:$ Percentage change in 2018Q4, results are presented as percentage differences compared to the counterfactual (extrapolation of preannouncement trend)

*** $p<0.001, * \star p<0.01, * p<0.05$. Standard errors in parentheses.

Network measures were normalized by adjusting for the panel size of networks. 


\begin{tabular}{|c|c|c|c|c|c|c|c|}
\hline Outcomes & $\begin{array}{l}\text { Threshold } \\
\text { (No. of } \\
\text { shared } \\
\text { patients) }\end{array}$ & $\begin{array}{l}\text { Baseline } \\
\text { level }\end{array}$ & $\begin{array}{l}\text { Baseline } \\
\text { trend }\end{array}$ & $\begin{array}{l}\text { Post- } \\
\text { implementation } \\
\text { level change }\end{array}$ & $\begin{array}{l}\text { Post- } \\
\text { implementation } \\
\text { trend change }\end{array}$ & $\begin{array}{l}\text { Absolute } \\
\text { difference } \\
\text { in } \\
2018 Q 4 \\
(95 \% \mathrm{Cl})^{+}\end{array}$ & $\begin{array}{l}\text { Percentage } \\
\text { change in } \\
2018 \mathrm{4} 4 \\
(95 \% \mathrm{Cl})^{\ddagger}\end{array}$ \\
\hline & & & & & & $\begin{array}{l}(2.497 \text { to } \\
4.411)\end{array}$ & $\begin{array}{l}(84.0 \text { to } \\
252.5)\end{array}$ \\
\hline \multicolumn{8}{|c|}{$\begin{array}{l}\text { t: Absolute difference in } 2018 \mathrm{Q} 4 \text {, the difference between the observed outcome and the counterfactual } \\
\text { (extrapolation of pre-announcement trend) }\end{array}$} \\
\hline \multicolumn{8}{|c|}{$\begin{array}{l}\text { ‡: Percentage change in } 2018 Q 4 \text {, results are presented as percentage differences compared to the counterfactual } \\
\text { (extrapolation of preannouncement trend) }\end{array}$} \\
\hline \multicolumn{8}{|c|}{$\star \star \star * p<0.001,{ }^{* *} p<0.01, * p<0.05$. Standard errors in parentheses. } \\
\hline
\end{tabular}

\section{Discussion}

This study is the first to investigate the empirical impacts of the HMS policy on the volume of patient encounters and local physician networks. Our results confirmed the expectation that the HMS policy was effective in redirecting patient flows to PCFs and promoting the centrality of PCPs within their professional network, indicating a better coordinated and integrated care for patients with hypertension in Yinzhou district, Ningbo, China.

As the cornerstone of the HMS policy package, primary care gatekeeping might account for most of the increased patient volumes at PCFs and enhanced centrality of PCPs in healthcare delivery as observed from our results. Patients were incentivized to sign a contract with a family doctor in primary care facilities and used the family doctor as the first person of contact within Yinzhou referral system. Gatekeeping promoted PCP-coordinated care services and thus integration of care delivery across tiers as family doctors maintained close working relationships with $[22,40]$ professionals at higher-tier hospitals[4].[41, 42] Family doctors also facilitated the integration of services at the primary care level led a multidisciplinary team to provide comprehensive services. The team included a family doctor, a nurse, a public health specialist, and so forth. Family doctors led the development of team priorities and treatment choices that adhered to clinical protocols and coordinated team members to provide comprehensive care. Vertical and horizontal integration of care services altogether relied on PCP's role as a gatekeeper and contributed to better integration of care and thus patient outcomes.

Medical consortiums could be another reason for the positive association between the HMS policy and increased patient volumes observed at PCFs. Previous studies suggested that facility and practitioner capacity and drug availability were critical factors influencing patients' choices of healthcare facility in China, and that $[22,43,44]$ establishing medical consortiums proved an effective strategy to strengthen PCF capacity and likability.[41] Yinzhou deployed four strategies to encourage the establishment of medical consortiums in Yinzhou. First, hospitals and PCFs previously used different electronic information systems and providers at each could only access patient records within their own system. After the HMS policy, the local electronic medical records systems were integrated and made accessible to all healthcare providers of the same medical consortium. Specialist physicians at tiered hospitals could thus provide timely technical assistance to PCPs online. Second, each health medical facility used to adhere to different restrictions on dispensable drugs according to its tier classification. As in most cases in China, PCFs might not be able to dispense the medicine that a patient needed. Indeed, research showed that around one-third of patients 
attended higher-tier hospitals solely to obtain drugs, which exacerbated overcrowding at secondary and tertiary hospitals and contributed to tension between providers and patients.[3, 4] The HMS policy loosened these restrictions and allowed drugs to be shared within a medical consortium. Residents could thus access adequate supplies of drugs at PCFs of close proximity to them and waived the necessity of visiting hospitals. Third, the HMS policy also encouraged experienced physicians at higher-tier hospitals to serve in PCFs in the same medical consortium, which promoted communications among physicians across tiers and strengthened PCP and PCF capacity.[41] Fourth, the HMS package provided standardized further education and regular medical training sessions for PCPs to strengthen their professional profile and capacity to provide health services of quality.[22, 41, 45] However, it could take years for capacity strengthening strategies to drive health system improvements and hence we did not observe short-term effects immediately after the HMS policy. These might justify most of the significant trend changes and insignificant level changes in this study.

Gradient reimbursement schemes also helped to achieve the goal of the HMS policy of influencing residents' healthcare-seeking behaviors and directing patient flows to PCFs. Reimbursement rates for inpatients through family doctor's referral were higher than for non-referred cases, and a reimbursement gap for medical deductibles was set between different tiers of medical institutions. This echoed an earlier study that adjustments in health insurance reimbursement can promote patient visits to PCFs.[22] As Out-of-pocket medical expenses are one of the most determining factor influencing health-seeking behaviors of Chinese patients, $[9,20]$ studies observed that decreasing user fees for primary care and raising user fees for hospital ambulatory care increased primary care utilization.[15, 46, 47]

Although the HMS policy in the Yinzhou model has been successfully implemented, many other pilots in China were not led by PCFs and PCPs remained peripheral within professional networks.[3] Some studies observed limited effects of the gatekeeping policy in strengthening primary care and questioned the effectiveness of gatekeeping as a standalone strategy to improve system efficiency and provide quality care.[15] In Jilin, a province in northeastern China, patients' first contact to PCPs decreased by $19.80 \%$ in urban areas and $12.80 \%$ in rural areas between 2013 and 2018 , despite the local gatekeeping program to redirect patient flows to PCFs.[44] We reasoned two unique factors which distinguished the Yinzhou model from previous pilots. First, Yinzhou's pilot provided financial incentives for PCPs to encourage behavior change. A fixed fee of $150 \mathrm{CNY}$ per resident per year was offered to PCPs if they signed a family doctor contract.[3, 48] Second, as mentioned above (appendix 2), the Yinzhou model did not rely solely on gatekeeping but issued three intertwined policy actions that called for cross-sectional collaborations. Primary care gatekeeping was embedded in the capacity-building strategy to establish medical consortiums and incentivized by gradient reimbursement schemes. Intertwined and cross-sectional efforts ensured that patients with complex diseases were transferred to higher-tier hospitals for advanced treatment while those with chronic diseases or minor conditions were transferred to PCFs for routine care in a timely and coordinated manner.[22, 41] As inferred from this paper and verified by a growing body of research and practices, primary care keeping alone cannot guarantee effective system change. Enhanced health system efficiency, quality of care, and population health require cross-sectional collaboration and constant policy investments.

Our results also showed that the growth of PCP degree was less than that of PCP patient encounter ratio at 2018Q4 ( $23.6 \%$ vs $42.7 \%)$ and that the average degree of all physicians decreased from 29.3 to 27.9 after the introduction of the HMS policy. These results imply that the HMS policy regulated health-seeking behaviors and reduced disorderly patient flow. However, they can also imply that referrals only happened within each medical consortium and institutional challenges remained for patients who might need to be transferred across alliances. The leading hospital may take advantage of its role within the medical consortium to increase service volumes for revenues, rather than 
improving patient health outcomes.[4] The monopoly of patient sources may compromise hospital competition and thus quality of care, representing a caution to consider when designing future interventions.[41, 49]

This study has several limitations. First, we did not adjust for physician-level confounding which could have contributed to some associations we observed in the ITS model. However, this study aimed at using a data-driven approach to reproduce time trends observed in China; isolating the independent effect of the HMS policy on a physician adjusted for physician-level characteristics was beyond the aim of this study. Admittedly, the magnitude of our results is unlikely to be generalizable to other jurisdictions in China considering implementing the HMS policy. Second, the lack of a proper geographical comparison region as a control series limited our ability to control for external factors. To mitigate this limitation, we ensured that there were no other contextual factors that might alter our outcome measures during the study period by interviewing local stakeholders. We also conducted a sensitivity analysis by bringing forward the occurrence time of the HMS policy and confirmed that there were no other motivating factors. Third, although using the patient-sharing technique to construct physician networks has been validated by literature,[28] we did not have data on what information or behaviors were passed on through patient-sharing relationships between physicians. To assure that we retained the most important patient-sharing links, we took the following actions: we only included patients with hypertension to construct a more homogeneous cohort and networks to PCPs as well as medical specialists; we excluded patient encounters generated from physicians who practiced outside of Yinzhou district; we chose different thresholds (i.e., the number of shared patients) to identify a valid relationship and construct a robust model. Finally, because the study was limited to patients with hypertension, we could not examine outcomes within subsets of the population or patients with other chronic diseases.

Implications for practice and research

Our findings provide empirical evidence on the impact of the HMS policy and can provide some implications for future policy and program development. We observed that the HMS policy has lasting impacts on local health service provision after implementation (and the effects tend to grow with time), policymakers should thus sustain HMS policy efforts to obtain long-term and large-scale benefits. It's important to note that, despite the favorable effects of the HMS policy shown in this study, the Yinzhou model is far from a panacea, especially for patients who need to be transferred across medical consortiums. A provider payment overhaul would be necessary to reorient profit-driven hospitals towards maximizing population health gains. A more effective incentive mechanism among medical consortiums should be developed. Moreover, future policies should construct a performance evaluation system and performance-based feedback loops to identify system inefficiencies and malfunctions. The evaluation results can also be used to design physician rewards for improved primary care and chronic disease management. The impact of the HMS policy on service utilization, costs, and quality and outcomes of care will be explored as part of our ongoing evaluations.

\section{Conclusion}

To our best knowledge, this is the first empirical study to evaluate the impact of the HMS policy on patient encounters in a middle-income country, extending our understandings of this policy with social network analysis. The findings can provide some references to other countries without a mandatory gatekeeping system. The HMS policy package of Yinzhou is effective to incentivize patients to visit PCFs and enhance the centrality of PCPs within their health care delivery networks. These changes have profoundly reshaped the local health system and healthcare delivery.

Resources, technology and staff should be further allocated to sustain and promote the implementation of the HMS policy.

Page 12/19 


\section{Abbreviations}

\begin{tabular}{ll} 
SNA & social network analysis \\
\hline PCPs & primary care physicians \\
\hline LMIC & low- and middle-income countries
\end{tabular}

\section{Declarations}

\section{Ethics approval and consent to participate}

Not applicable.

\section{Consent for publication}

Not applicable.

\section{Availability of data and materials}

All data generated or analyzed during this study are included in this published article and its electronic supplementary material.

\section{Competing interests}

All authors declare that they have no conflicts of interest.

\section{Funding}

This work was supported by National Natural Science Foundation of China (NSFC: 72074007)

\section{Authors' contributions}

$\mathrm{HH}$ was a major contributor in writing the manuscript. $\mathrm{RW}$ and $\mathrm{HH}$ analyzed and visualized the social network data. $\mathrm{HL}$ reviewed and edited the manuscript. $\mathrm{SH}$ and $\mathrm{HL}$ contributed to data curation and supervised the data usage. The study idea originated from $\mathrm{HH}$ and was further developed in discussions with XG and LS and finalized in communication with all authors. All authors contributed to the submitted version and approved the final manuscript. $X G$ is the overall guarantor for the manuscript.

\section{Acknowledgements}

The authors are very grateful for the valuable comments and suggestions of the anonymous reviewers.

\section{References}

[1] Wang X, Sun X, Gong F, Huang Y, Chen L, Zhang Y, et al. The Luohu Model: A Template for Integrated Urban Healthcare Systems in China. Int J Integr Care. 2018;18(4):3. 
[2] Supper I, Catala O, Lustman M, Chemla C, Bourgueil Y, Letrilliart L. Interprofessional collaboration in primary health care: a review of facilitators and barriers perceived by involved actors. J Public Health (Oxf). 2015;37(4):716-27.

[3] Yip W, Hsiao W. Harnessing the privatisation of China's fragmented health-care delivery. Lancet. 2014;384(9945):805-18.

[4] Wang X, Sun X, Birch S, Gong F, Valentijn P, Chen L, et al. People-centred integrated care in urban China. Bull World Health Organ. 2018;96(12):843-52.

[5] Chen F, Jiang Q, Lu Z, Cao S. General practitioners' perspectives of the integrated health care system: a crosssectional study in Wuhan, China. Fam Pract. 2021;38(2):103-8.

[6] Liang D, Mei L, Chen Y, Zhou P, Yang X, Huang J. Building a People-Centred Integrated Care Model in Urban China: A Qualitative Study of the Health Reform in Luohu. Int J Integr Care. 2020;20(1):9.

[7] Rittenhouse DR, Shortell SM, Fisher ES. Primary care and accountable care--two essential elements of deliverysystem reform. N Engl J Med. 2009;361(24):2301-3.

[8] Leichsenring K. Developing integrated health and social care services for older persons in Europe. Int J Integr Care. 2004;4:e10.

[9] Jing X, Xu L, Qin W, Zhang J, Lu L, Wang Y, et al. The Willingness for Downward Referral and Its Influencing Factors: A Cross-Sectional Study among Older Adults in Shandong, China. Int J Environ Res Public Health. 2020;17(1).

[10] Zeng Y, Xu W, Chen L, Chen F, Fang Y. The Influencing Factors of Health-Seeking Preference and Community Health Service Utilization Among Patients in Primary Care Reform in Xiamen, China. Patient Prefer Adherence. 2020;14:653-62.

[11] Wang Y, Sun L, Hou J. Hierarchical Medical System Based on Big Data and Mobile Internet: A New Strategic Choice in Health Care. JMIR Med Inform. 2017;5(3):e22.

[12] Xiao Y, Chen X, Li Q, Jia P, Li L, Chen Z. Towards healthy China 2030: Modeling health care accessibility with patient referral. Soc Sci Med. 2021;276:113834.

[13] Bodenheimer T, Wagner EH, Grumbach K. Improving primary care for patients with chronic illness. Jama. 2002;288(14):1775-9.

[14] Briggs AM, Valentijn PP, Thiyagarajan JA, Araujo de Carvalho I. Elements of integrated care approaches for older people: a review of reviews. BMJ Open. 2018;8(4):e021194.

[15] Xu J, Powell-Jackson T, Mills A. Effectiveness of primary care gatekeeping: difference-in-differences evaluation of a pilot scheme in China. BMJ Glob Health. 2020;5(8).

[16] Lu C, Zhang Z, Lan X. Impact of China's referral reform on the equity and spatial accessibility of healthcare resources: A case study of Beijing. Soc Sci Med. 2019;235:112386.

[17] Du X, Patel A, Anderson CS, Dong J, Ma C. Epidemiology of Cardiovascular Disease in China and Opportunities for Improvement: JACC International. J Am Coll Cardiol. 2019;73(24):3135-47. 
[18] Yip W, Fu H, Chen AT, Zhai T, Jian W, Xu R, et al. 10 years of health-care reform in China: progress and gaps in Universal Health Coverage. Lancet. 2019;394(10204):1192-204.

[19] Yang G, Wang Y, Zeng Y, Gao GF, Liang X, Zhou M, et al. Rapid health transition in China, 1990-2010: findings from the Global Burden of Disease Study 2010. Lancet. 2013;381(9882):1987-2015.

[20] Tao T, Hou L, Shao R. Factors associated with the choice of primary treatment at the community level among the middle-aged and elderly in China. Int J Health Plann Manage. 2021;36(2):442-58.

[21] Yang N, Shen L, Shu T, Liao S, Peng Y, Wang J. An integrative method for analyzing spatial accessibility in the hierarchical diagnosis and treatment system in China. Soc Sci Med. 2021;270:113656.

[22] Zhou Z, Zhao Y, Shen C, Lai S, Nawaz R, Gao J. Evaluating the effect of hierarchical medical system on health seeking behavior: A difference-in-differences analysis in China. Soc Sci Med. 2021;268:113372.

[23] Huang K, Tao S, Zhou X, Mo J, Zhu B, Shen P, et al. Incidence rates of health outcomes of interest among Chinese children exposed to selected vaccines in Yinzhou Electronic Health Records: A population-based retrospective cohort study. Vaccine. 2020;38(18):3422-8.

[24] Lin H, Tang X, Shen P, Zhang D, Wu J, Zhang J, et al. Using big data to improve cardiovascular care and outcomes in China: a protocol for the CHinese Electronic health Records Research in Yinzhou (CHERRY) Study. BMJ Open. 2018;8(2):e019698.

[25] Wagner AK, Soumerai SB, Zhang F, Ross-Degnan D. Segmented regression analysis of interrupted time series studies in medication use research. J Clin Pharm Ther. 2002;27(4):299-309.

[26] Barnett ML, Landon BE, O'Malley AJ, Keating NL, Christakis NA. Mapping physician networks with self-reported and administrative data. Health Serv Res. 2011;46(5):1592-609.

[27] Landon BE, Keating NL, Barnett ML, Onnela JP, Paul S, O'Malley AJ, et al. Variation in patient-sharing networks of physicians across the United States. Jama. 2012;308(3):265-73.

[28] DuGoff EH, Fernandes-Taylor S, Weissman GE, Huntley JH, Pollack CE. A scoping review of patient-sharing network studies using administrative data. Transl Behav Med. 2018;8(4):598-625.

[29] Glegg SMN, Jenkins E, Kothari A. How the study of networks informs knowledge translation and implementation: a scoping review. Implement Sci. 2019;14(1):34.

[30] Uddin S, Kelaher M, Srinivasan U. A framework for administrative claim data to explore healthcare coordination and collaboration. Aust Health Rev. 2016;40(5):500-10.

[31] Bavelas, Alex. Communication Patterns in Task-Oriented Groups. The Journal of the Acoustical Society of America. 1950;22(6):725-30.

[32] Linton, C., Freeman. Centrality in social networks conceptual clarification. Social Networks. 1978.

[33] Uddin S, Hossain L, Kelaher M. Effect of physician collaboration network on hospitalization cost and readmission rate. Eur J Public Health. 2012;22(5):629-33. 
[34] Everett M, Borgatti S. The Centrality of Groups and Classes. Journal of Mathematical Sociology. 1999;23:181201.

[35] Uddin MS, Hossain L. Social networks enabled coordination model for cost management of patient hospital admissions. J Healthc Qual. 2011;33(5):37-48.

[36] Barnett ML, Christakis NA, O'Malley J, Onnela JP, Keating NL, Landon BE. Physician patient-sharing networks and the cost and intensity of care in US hospitals. Med Care. 2012;50(2):152-60.

[37] Casalino LP, Pesko MF, Ryan AM, Nyweide DJ, Iwashyna TJ, Sun X, et al. Physician Networks and Ambulatory Care-sensitive Admissions. Med Care. 2015;53(6):534-41.

[38] Newey WK, West KD. A SIMPLE, POSITIVE SEMI-DEFINITE, HETEROSKEDASTICITY AND AUTOCORRELATION CONSISTENT COVARIANCE MATRIX. Econometrica (1986-1998). 1987;55(3):703.

[39] Guido, W., Imbens, and, Thomas, Lemieux. Regression discontinuity designs: A guide to practice. Journal of Econometrics. 2008.

[40] Rice T, Rosenau P, Unruh LY, Barnes AJ, Saltman RB, van Ginneken E. United States of America: health system review. Health Syst Transit. 2013;15(3):1-431.

[41] Cai M, Liu E, Tao H, Qian Z, Fu QJ, Lin X, et al. Does A Medical Consortium Influence Health Outcomes of Hospitalized Cancer Patients? An Integrated Care Model in Shanxi, China. Int J Integr Care. 2018;18(2):7.

[42] Qian Y, Hou Z, Wang W, Zhang D, Yan F. Integrated care reform in urban China: a qualitative study on design, supporting environment and implementation. Int J Equity Health. 2017;16(1):185.

[43] Liu Y, Zhong L, Yuan S, van de Klundert J. Why patients prefer high-level healthcare facilities: a qualitative study using focus groups in rural and urban China. BMJ Glob Health. 2018;3(5):e000854.

[44] Liao R, Liu Y, Peng S, Feng XL. Factors affecting health care users' first contact with primary health care facilities in north eastern China, 2008-2018. BMJ Glob Health. 2021;6(2).

[45] Wu D, Lam TP, Lam KF, Zhou XD, Sun KS. Health reforms in china: the public's choices for first-contact care in urban areas. Fam Pract. 2017;34(2):194-200.

[46] Liu X, Xu J, Yuan B, Ma X, Fang H, Meng Q. Containing medical expenditure: lessons from reform of Beijing public hospitals. Bmj. 2019;365:I2369.

[47] Shen M, He W, Li L. Incentives to use primary care and their impact on healthcare utilization: Evidence using a public health insurance dataset in China. Soc Sci Med. 2020;255:112981.

[48] Struijs JN, Baan CA. Integrating care through bundled payments-lessons from The Netherlands. N Engl J Med. 2011;364(11):990-1.

[49] Cooper Z, Gibbons S, Jones S, McGuire A. Does Hospital Competition Save Lives? Evidence from the English NHS Patient Choice Reforms. Econ J (London). 2011;121(554):F228-f60.

\section{Figures}




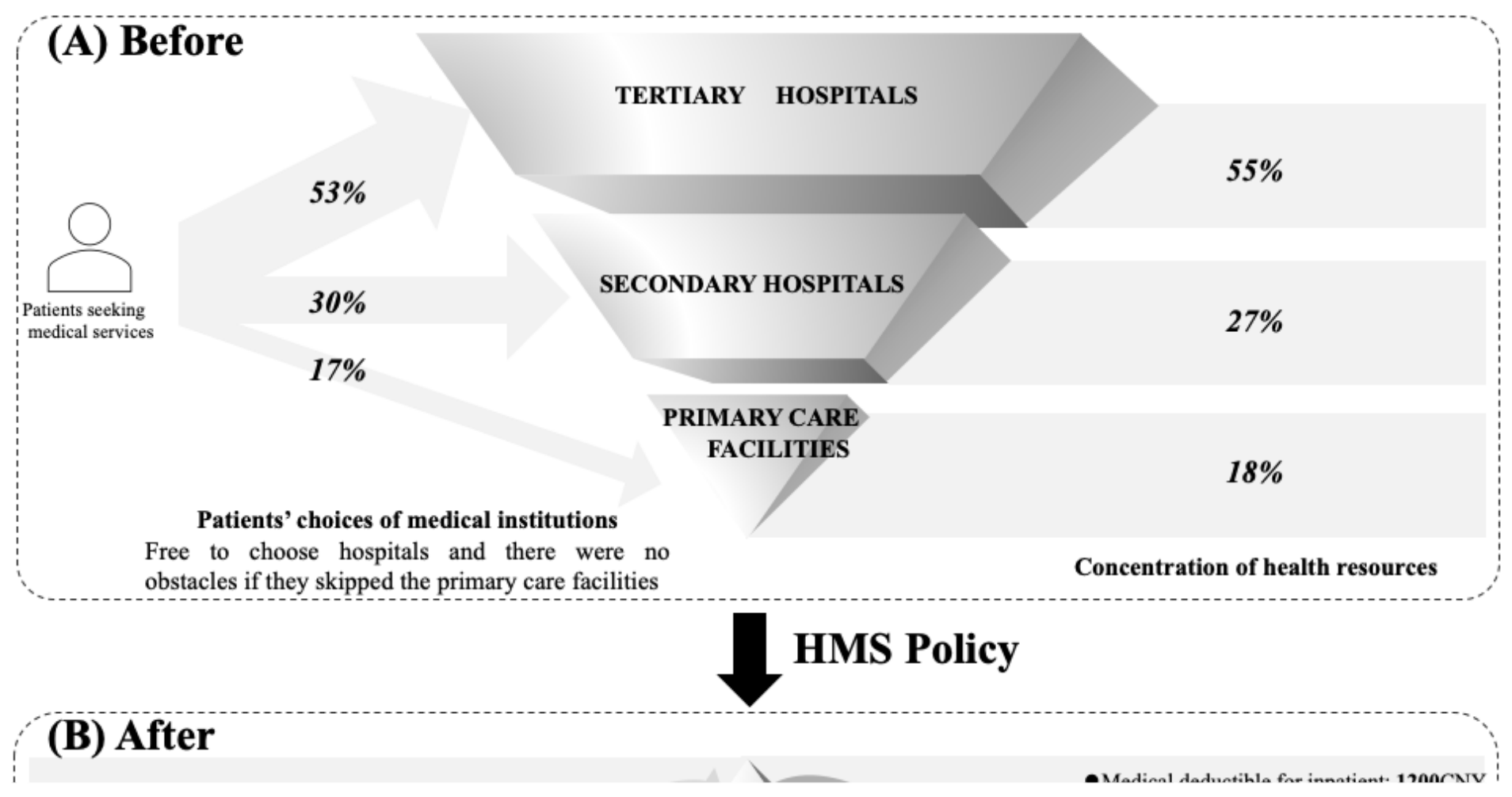

Figure 1

Framework of hierarchical medical system (HMS) policy to achieve PCP-based integrated care in Yinzhou district, Ningbo city Note: total revenue in each group, extracted from China health statistical yearbook 2015, is used for computing the concentration of health resources before the HMS policy. Patients' choices of hospitals before the reform are extracted from the 2012-2015 Health and Family Planning Development Statistics Bulletin in a city, China 


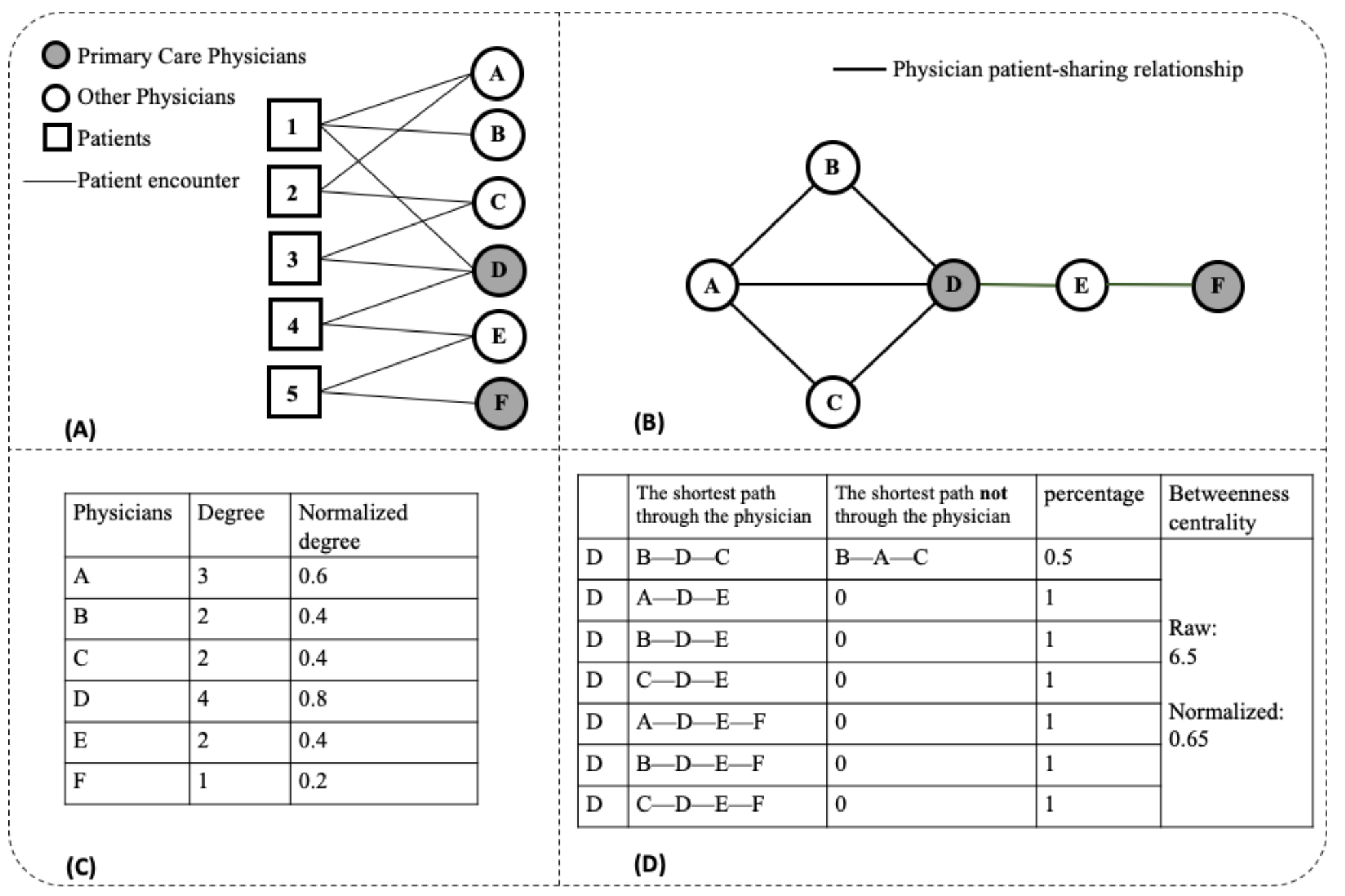

\section{Figure 2}

Illustrative example of the construction of a physician network from patient encounter data. Notes: (A) illustrates how patients create links among physicians. (B) a group of patient-physician ties depicted in Figure 2A are transformed into a simplified format that does not include designated patients. (C) shows each physician's degree. Degree is the number of connections incident to a given physician, or equivalently, the number of other physicians an individual physician was connected with through the sharing of patients. Normalization is performed by dividing the raw degree by $n-1$, where $n$ is the number of physicians in the graph. PCP degree ratio (i.e., the mean degree of the primary care physicians divided by the mean degree of all other physicians in the network) in this schematic illustration is 1.111 , meaning that the degree of PCP is nearly equal to the degree of other physicians. (D) presents the betweenness centrality of physician D. Betweenness centrality measures are aimed at summarizing the extent to which a node is located on the shortest paths between other pairs of nodes. This measure is normalized by multiplying the raw betweenness centrality by $2 /(n 2-3 n+2)$, and $n$ is the number of physicians in the graph. The betweenness centrality of physician $D$ is 6.5 (normalized: 0.65 ), and using the same method, the betweenness centrality of physician $A, B, C, E, F$ is 0.5 (normalized: 0.05 ), 0 (normalized: 0 ), 0 (normalized: 0 ), 4 (normalized: 0.4 ), and 0 (normalized: 0 ), respectively. PCP betweenness centrality ratio (i.e., the mean betweenness centrality of the primary care physicians divided by the mean betweenness centrality of all other physicians in the network) in this schematic illustration is 2.889 , meaning that PCPs in this network are on average about three times as central as other physicians. 

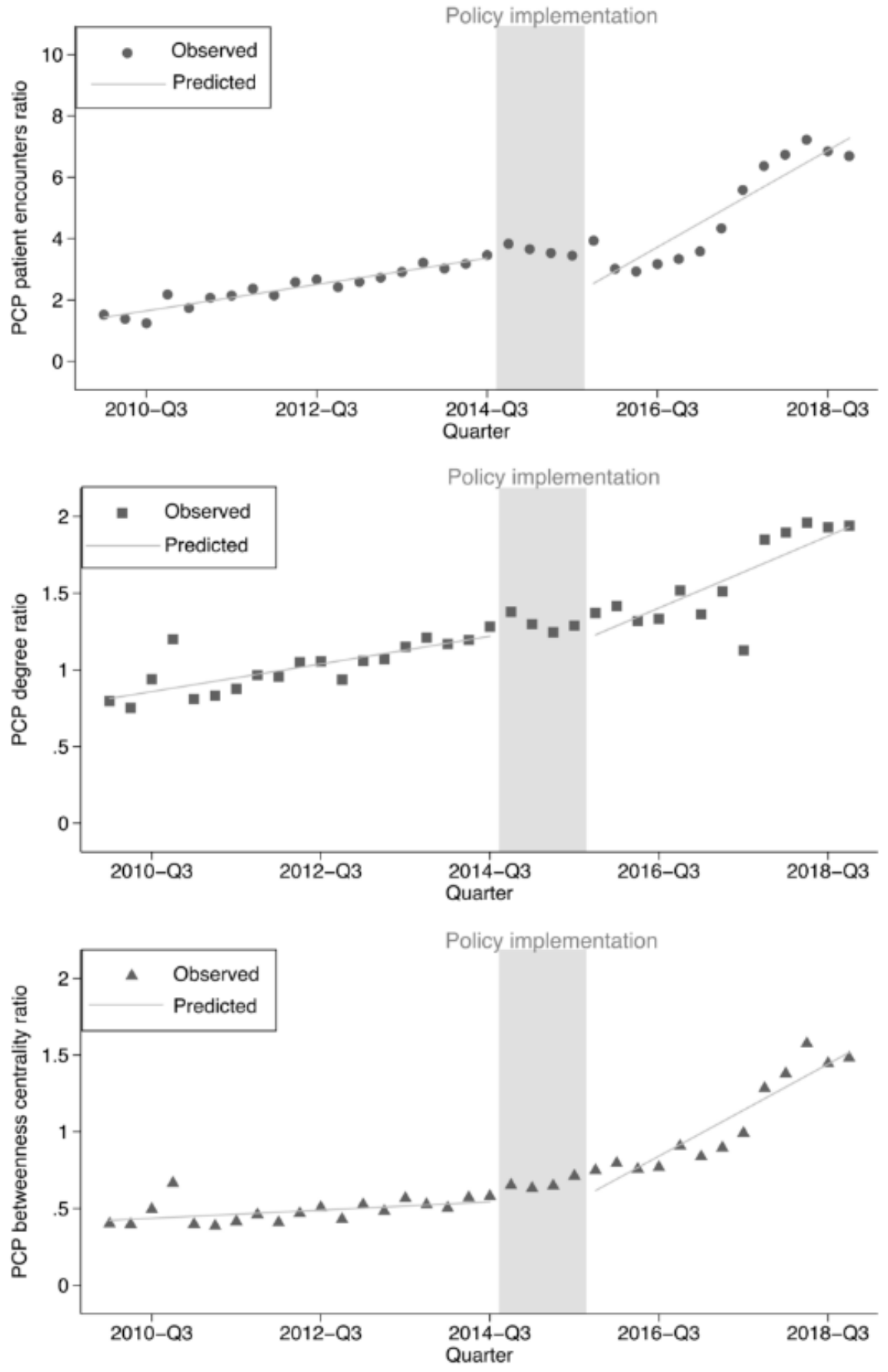

\section{Figure 3}

Results of ITS analyses of the impacts of the hierarchical Medical System (HMS) policy on PCP patient encounter ratio, PCP degree ratio, and PCP betweenness centrality ratio.

\section{Supplementary Files}

This is a list of supplementary files associated with this preprint. Click to download.

- STROBEchecklist1202.docx

- appendix20211202.pdf 